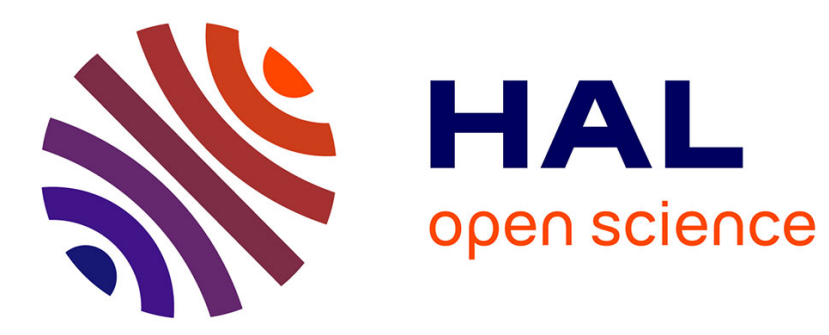

\title{
A new optimization approach for the calibration of an ultrasound probe using a 3D optical localizer
}

Guillaume Dardenne, Daniel Gil Cano, Chafiaa Hamitouche, Eric Stindel, Christian Roux

\section{- To cite this version:}

Guillaume Dardenne, Daniel Gil Cano, Chafiaa Hamitouche, Eric Stindel, Christian Roux. A new optimization approach for the calibration of an ultrasound probe using a 3D optical localizer. EMBS 2007 : 29th annual international conference of IEEE engineering in medecine and biology society, Aug 2007, Lyon, France. pp.3048 - 3051, 10.1109/IEMBS.2007.4352971 . hal-02926767

\section{HAL Id: hal-02926767 https://hal.science/hal-02926767}

Submitted on 1 Sep 2020

HAL is a multi-disciplinary open access archive for the deposit and dissemination of scientific research documents, whether they are published or not. The documents may come from teaching and research institutions in France or abroad, or from public or private research centers.
L'archive ouverte pluridisciplinaire HAL, est destinée au dépôt et à la diffusion de documents scientifiques de niveau recherche, publiés ou non, émanant des établissements d'enseignement et de recherche français ou étrangers, des laboratoires publics ou privés. 


\title{
A new optimization approach for the calibration of an ultrasound probe using a 3D optical localizer
}

\author{
G. Dardenne ${ }^{1,2,3}$, J.D. Gil Cano ${ }^{1,2}$, C. Hamitouche ${ }^{1,2}$, E. Stindel ${ }^{1,4}$ and C. Roux ${ }^{1,2}$ \\ ${ }^{1}$ INSERM, U650, Brest, F-29200 France ; \\ ${ }^{2}$ ENST Bretagne, GET-ENST, Brest, F-29200 France ; \\ ${ }^{3}$ Université de Bretagne Occidentale, Brest, F-29200 France ; \\ ${ }^{4}$ CHU Brest, Service d'Orthopédie-Traumatologie, Brest, F-29200 France.
}

\begin{abstract}
This paper describes a fast procedure for the calibration of an ultrasound (US) probe using a 3D optical localizer. This calibration step allows us to obtain the 3D position of any point located on the $2 D$ ultrasonic (US) image. To carry out correctly this procedure, a phantom of known geometric properties is probed and these geometries are found in the US images. A segmentation step is applied in order to obtain automatically the needed information in the US images and then, an optimization approach is performed to find the optimal calibration parameters. A new optimization method to estimate the calibration parameters for an ultrasound (US) probe is developed.
\end{abstract}

Index Terms - Calibration, Genetic algorithms, Convergence of numerical methods, Ultrasound imaging, Image processing.

\section{INTRODUCTION}

Several methods have been reported and a set of phantoms (cross-wire, single-wall, Cambridge phantom,...) have been used to perform the calibration of an US probe [1]. In our application concerning the navigated orthopaedic surgery, we wish to integrate an echograph to fill the lack of information on patient morphology [2]. Therefore, several constraints must be taken into account : accuracy, simplicity of implementation, reproducibility, robustness and rapidity. Our choice is then the single-wall procedure. This calibration procedure is based on a navigation station (Surgetics ${ }^{\circledR}$ station) composed of two cameras, of reflective markers and of an US probe. The use of this kind of phantom has the main advantage to provide very clear lines on US images and therefore, a simple image processing method can be used to detect the lines on these images. A new optimization method is then carried out to estimate the optimal calibration parameters. This new approach allows us to obtain a global convergence to the optimal calibration parameters without a priori knowledge on the US probe's geometry. Once all the US images are acquired and the estimation step is done, the accuracy of the calibration procedure is estimated. The known position of a point in the space is, therefore, compared with its position found with the US images and the calibration. A good accuracy of the estimation of the different calibration parameters is crucial because the quality of operations carried out thanks to the ultrasound and the calibration such as obtaining a volume or a position of an object depends directly on the calibration procedure.
Therefore, this step is a key stage which must be carried out carefully.

\section{THEORETICAL ASPECTS}

\section{A. Background}

The problem of calibration can be expressed with the following equation [3] :

$$
\vec{x}_{\text {probe }}=T_{R} \times S \times \vec{x}_{\text {image }}
$$

where :

- $\vec{x}_{\text {image }}$ represents the coordinates of a point in the US image and is equal to : $\left[\begin{array}{llll}x & y & 0 & 1\end{array}\right]^{T}$.

- $S$ represents the scale factors matrix and is equal to :

$$
S=\left[\begin{array}{cccc}
s_{x} & 0 & 0 & 0 \\
0 & s_{y} & 0 & 0 \\
0 & 0 & 1 & 0 \\
0 & 0 & 0 & 1
\end{array}\right]
$$

with $s_{x}$ and $s_{y}$ the scale factors on $x$ and $y$.

- $T_{R}$ represents the rigid matrix transformation between the image reference and the probe reference and is equal to :

$$
T_{R}=\left[\begin{array}{cc}
R(\alpha, \beta, \gamma) & T\left(t_{x}, t_{y}, t_{z}\right) \\
0 & 1
\end{array}\right]
$$

with $R$ the $3 \times 3$ matrix representing the rotations and $T$ the translations.

The aim of the calibration is therefore to estimate the three rotation parameters $\alpha, \beta, \gamma$, the three translation $t_{x}, t_{y}, t_{z}$ ones and the two scale factors $s_{x}$ and $s_{y}$ of the transformation between the probe referential and the image referential (see Fig.1). This transformation will allow us to obtain the 3D position of any points in the 2D US images. To carry out the calibration, a plane is probed. The lines representing the plane in the US images are then detected and the eight parameters are estimated using a new optimization procedure. 


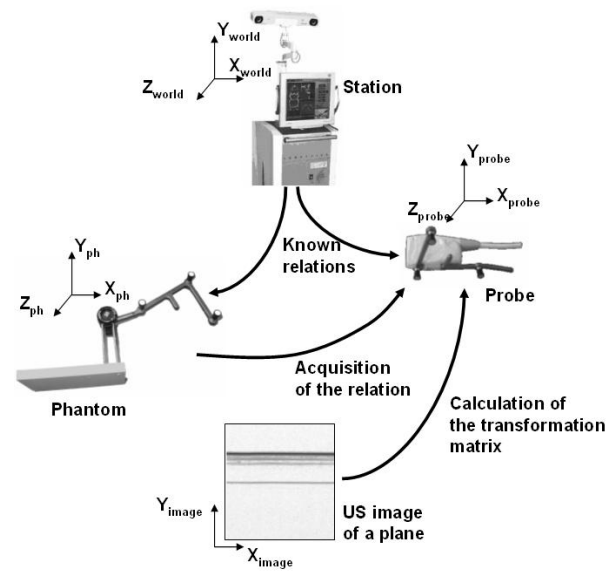

Fig. 1. General scheme for the calibration of an US probe.

\section{B. Segmentation step : detection of the lines in US images}

To obtain the useful information in the US image, a processing step is needed. In our case, the line representing the plane in the US image have to be detected (Fig. 2). The first step of the processing have to clean the images and preserve the useful information. Thus, a threshold is used to limit the noise, and then, a gradient operation is carried out to obtain the edges. The line is then detected using the Hough transform [4]. This operation consists in representing a point located in the US image to a curve in the Hough space thanks to the following equation :

$$
x \times \cos (\theta)+y \times \sin (\theta)-\rho=0
$$

where $(x, y)$ are the coordinates of the point in the US image and $(\rho, \theta)$ the coordinates in the Hough space. A Hough property shows that if a line is represented in an image, all the points of this line are transformed into curves in the Hough space which are concurrent in a same point $\left(\rho_{0}, \theta_{0}\right)$. Thus, this point must be detected with a high accuracy because it represents the needed information. For that, the

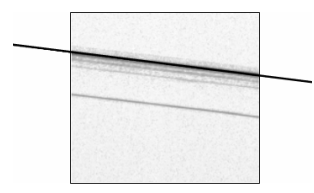

Fig. 2. US image of the plane and detection.

accumulator principle is used. Each curve in the Hough space has the same intensity as its point in the US image. The accumulator principle adds on each point in the Hough space the intensity of the different curves passing through this point. Then, several points with the highest intensities are preserved in the Hough space for more robustness and the one which has the highest $y$ coordinate in the US image is taken (there is no reflexion actually in the US image above the line, therefore, the line representing the plane is the highest line i.e. the highest $y$ coordinate : see Fig.2). This last point describes the position of the line representing the plane in the US image.

\section{The optimization step : estimation of the calibration parameters}

This step allows us to estimate the eight parameters (three rotations, three translations and two scale factors) by minimizing the distance between the real plane and the points obtained with the US images. It is therefore equivalent to minimizing the following equation [5] in the probe referential (Fig. 1) :

$$
f\left(t_{x}, t_{y}, t_{z}, \alpha, \beta, \gamma, s_{x}, s_{y}\right)=\frac{1}{2} \sum_{i=1}^{N} d\left(\pi, M_{i}\right)^{2}
$$

where

- $\pi$ is the plane of known position.

- $M_{i}$ represents the different points of the line obtained with the US images.

- $d\left(\pi, M_{i}\right)$ is the euclidean distance between the plane and the different points in the probe referential and is equal to :

$$
d\left(\pi, M_{i}\right)=a X_{i}+b Y_{i}+c Z_{i}+d
$$

with $a, b, c$ and $d$ the known parameters of the plane and $X_{i}, Y_{i}$ and $Z_{i}$ the coordinates of the point $M_{i}$ in the probe referential.

Two different optimization approaches can be used to determine the calibration parameters :

- the iterative method.

- the closed-form (non-iterative) method.

However, concerning the wall-phantom, only the first method can be used because it is not possible to find the exact equation of the line defined by the intersection of the image plane and the wall phantom [1]. Therefore, the LevenbergMarquardt (LM) algorithm will be used. This iterative algorithm can locate the local minimum of a function that is expressed as the sum of squares of nonlinear function [6] [7]. To do that, the eight parameters need to be firstly initialized to begin the optimization. The LM algorithm is applied until the cost function representing the distance in the equation (4) is lower than a threshold : the calibration parameters are then found. Nevertheless, the initialization step is very important because the results can be false due to local convergence. A good initialization near the global minimum must be obtained to initialize correctly the LM algorithm. A method allowing us to obtain a good initialization have to be found in order to obtain correct calibration parameters whatever the US probe. A stochastic method, based on the genetic algorithms, is thus used.

\section{MATERIAL AND GENETIC ALGORITHMS}

\section{A. Material used}

Our method consists to image a plane with the US probe in different position in the space in order to obtain the calibration parameters. This procedure is based on a navigation system (Surgetics ${ }^{\circledR}$ station) which uses stereoscopic infrared cameras : two cameras emit infrared signals and receive those sent back by retro reflective markers situated on the 
references that are fixed on the objects we want to track. These trackers are called rigid bodies. Two types of trackers can be distinguished (fig. 3) : reference trackers and pointer tracker which is used to digitize the 3D targeted structure. Two rigid bodies are fixed on the US probe and on the plane

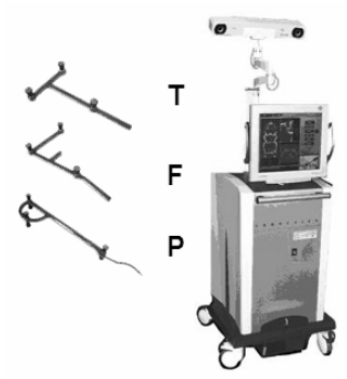

Fig. 3. Navigation station (right) and rigid bodies (left).

allowing us to know their positions and orientations in the space in real time.

\section{B. Basics of genetic algorithms}

As it has been seen before, the LM algorithm needs to be correctly initialized in order to obtain global convergence. A stochastic method, based on genetic algorithms, is then used to initialize this algorithm. This kind of method can detect global convergence in a search space but with not necessarily a good accuracy (fig. 4). The genetic algorithms are based
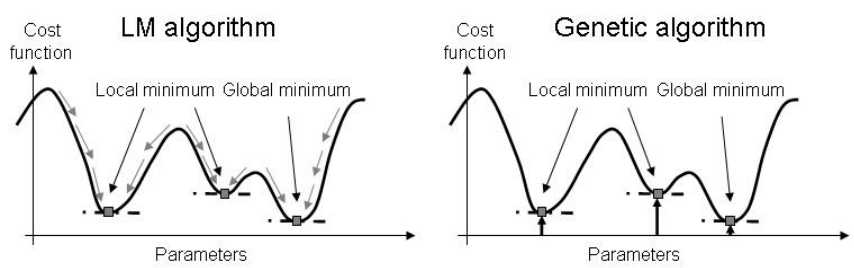

Fig. 4. Illustration of different optimization method in order to obtain a minimum.

on the Darwin theory which determines which individuals have to survive, which have to reproduce, which have to die. To do that, these algorithms are based on a group of genome or chromosome representing the different possible solutions. These genomes are evaluated thanks to an objective function in order to appraise its accuracy with regard to the final solution. Following this evaluation, the genome can survive, reproduce or die [8].

At the beginning of the algorithm, the genomes are chosen randomly in a search space and they evolve according to the Darwin theory. The genomes are, in general, brought progressively near the final solution. However, the final genome is an approximative value of the final solution and doesn't fall with high accuracy in the global minimum. The solution of the genetic algorithm is therefore used to initialize the LM algorithm which can locate the solution with a higher accuracy. The genetic algorithm have actually the advantage to find quickly in a search space a good initialization for an iterative technique. However, for a good working of the genetic algorithm, a good adjustment is required. To do that, a correct search space must be found, a good chromosome encoding is needed, a representative group of genome must be taken and enough transformation on theses genomes during the evolution of the algorithm like crossover or mutation must be carried out [9]. The genetic algorithm will stop when one of the considered criteria is reached (number of generation, value of the objective function, etc...).

\section{Use of genetic algorithms in the optimization step}

For a good working of the genetic algorithm in the optimization step of the calibration procedure, a search space must be firstly defined. To carry out correctly this definition, the US probe is digitized with the pointer tacker as described in the figure 5. This allows us to approximate the

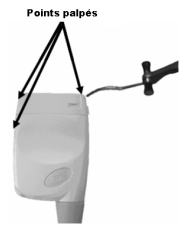

Fig. 5. Digitization of the probe.

transformation between the image and the probe. The search space for the genetic algorithm will be then the space around this approximation. Concerning the chromosome encoding, this one has eight genes (Fig. 6). Each gene corresponds to

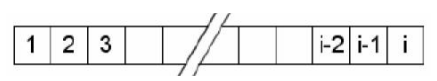

Fig. 6. Representation of a chromosome with i genes.

one parameter to find. Therefore there will be two genes for the scale factors, three genes for the rotation and three genes for the translation. A population of 200 genes is taken to be representative of the search space and a probability of 0.3 for the mutation and 0.7 for the crossover is carried out. These adjustments were found experimentally.

The genetic algorithms will stop when one of the following criteria is reached :

- the same score of the best chromosome is obtained 75 times in a row.

- the score of the best chromosome is under $1 \mathrm{~mm}$.

- the maximum number of generations is 150 .

These values are again found experimentally to obtain correct results as fast as possible. This optimization procedure has the main advantage to avoid physical measurements of the geometry between the rigid body attached on the US probe and the receptor in order to initialize correctly the LM algorithm and can therefore be used by any US probe without difficulties.

\section{RESULTS AND DISCUSSION}

\section{A. The operating speed of the procedure}

The operating speed of the procedure depends on different steps. The first step allows us to prepare the calibration of 
an US probe. The plane must be firstly digitized thanks to the pointer tracker in order to obtain the position and the orientation of the plane relative with its rigid body. The different parameters $a, b, c$ and $d$ of the plane are therefore estimated in the probe referential and are used to resolve the equation (4) in order to find the final solution. Then, the search space is defined for the genetic algorithm as described in the previous paragraph. This first procedure takes about two minutes. Then, three minutes are required to acquire and process the US images. Once all the images are processed, the optimization step takes about two minutes to estimate correctly the calibration parameters. In this step, the genetic algorithms take about 30 seconds and the LM algorithm lasts about 1,5 minutes. The speed of the LM algorithm become faster thanks to a method based on the multiresolution [10]. This technique allows us to increase the speed up to three or four times. In conclusion, the global procedure takes hardly ten minutes which is what we wanted.

\section{B. The accuracy of the calibration procedure}

The accuracy of the procedure for the calibration of an US probe is evaluated using a point of known position in the space. US images of this point are then obtained and the $T_{r}$ transformation is applied to know these coordinates in the probe referential. The position of the real point and the position of the point found thanks to US images are, after that, compared in the probe referential (table I).

To obtain a good evaluation of the accuracy, 40 calibration matrices are used. The accuracy of the system is given by the average of the 40 distances between the two points. The table I shows that the average accuracy is quite high

\begin{tabular}{|r||c|}
\hline Average accuracy $(\mathrm{mm})$ & 1.22 \\
\hline Std. Dev $(\mathrm{mm})$ & 0.42 \\
\hline Minimal Value $(\mathrm{mm})$ & 0.65 \\
\hline Maximal Value $(\mathrm{mm})$ & 2.44 \\
\hline
\end{tabular}

TABLE I

ACCURACY OF THE SYSTEM.

with 1,22 $\mathrm{mm}$ and a standard deviation of $0,42 \mathrm{~mm}$. This high standard deviation shows that the reproducibility of the procedure is not satisfying. This lack of reproducibility for the single wall phantom method is due to the beam thickness of the US probe [3] [11] (Fig. 7). When the probe is in

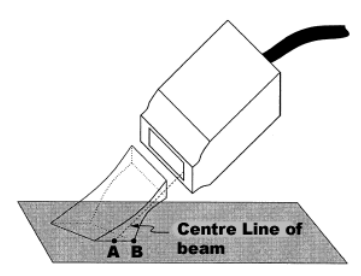

Fig. 7. Beam thickness problem in single wall calibration.

oblique position with regard to the plane the beam thickness can generate problems in the received image. The figure 7 shows that the point B is encountered by the US pulse before point $\mathrm{A}$ on the centerline and then, the echo from point $\mathrm{B}$ produces a response in the US image which does not reflect the true position of the wall. It's therefore very important to correct this lack of reproducibility using a phantom which avoids this beam thickness problem. Nevertheless, results obtained are in coherence with the literature. Concerning Prager [3] and Rousseau [10] who used the single wall phantom for the calibration, they obtained respectively an accuracy of $3.43 \mathrm{~mm}$ and $1.54 \pm 0.66 \mathrm{~mm}$ with the same procedure of evaluation. Concerning Prager, the calibration was carried out with an electromagnetic localizer whereas concerning Rousseau, it was carried with an optical localizer. It's therefore more appropriate to compare our results with those obtained with Rousseau.

\section{COnClusions}

In this paper, a rapid method with an easy set-up has been developed for the calibration of an US probe using a 3D optical localizer.

Concerning the optimization step of this procedure, a new method has been used which allows us to obtain a global solution in a defined space thanks to the stochastic behavior of the genetic algorithms. This method avoids a priori knowledge of the geometry of the US probe in order to initialize correctly the LM algorithm.

Concerning the accuracy of the procedure, very satisfying results have been obtained. The procedure lacks of reproducibility but a slight modification in the design of the calibration tool will allow us to improve the results. We will then have an efficient and automatic calibration procedure with the required accuracy and robustness, usable for clinical purposes.

\section{REFERENCES}

[1] L. Mercier, A review of calibration techniques for freehand 3-D ultrasound systems, Ultrasound in Med. \& Biol., vol. 31, 2005, pp 143-165.

[2] J. D. Gil Cano, "CT less based Computer Assisted Navigation System for High Tibial Osteotomy using an Optical Tracking System", Surgetica, 2002.

[3] R. W. Prager, Rapid calibration for 3-D freehand ultrasound, Ultrasound in Med. \& Biol., vol. 24, 1998, pp 855-869.

[4] F. Rousseau, "Robust and automatic calibration method for 3D freehand ultrasound", MICCAI, Montreal, 2003.

[5] F. Rousseau, "A fully automatic calibration procedure for freehand 3D ultrasound", IEEE International Symposium on Biomedical Imaging, Washington, 2002.

[6] I. A. Manolis, "A brief description of the Levenberg-Marquardt algorithm implemened by levmar", Foundation for research and technology, Heraklion, 2005.

[7] K. Madsen, "Methods for non-linear least squares problems", Technical University of Denmark, 2004.

[8] J. M. Rouet, Genetic algorithms for a robust 3-D MR-CT registration, IEEE Transactions on Information Technology in Biomedicine, vol. 4, 2000.

[9] M. Wall, "GAlib : a C++ library of genetic algorithm components", Mechanical Engineering Department, Massachusetts Institute of Technology, 1996.

[10] F. Rousseau, Méthodes d'analyse d'image et de calibration pour l'échographie $3 D$ en mode main libre, Ph.D. thesis, Université de Rennes I, 2003.

[11] R. W. Prager, "Automatic calibration for 3-D free-hand ultrasound", Cambridge university, 1997. 